\title{
Numerical simulations of impulsively excited magnetosonic waves in two parallel solar coronal slabs
}

\author{
R. Ogrodowczyk ${ }^{1}$ and K. Murawski ${ }^{2}$ \\ 1 Institute of Mathematics and Computer Sciences, The State University in Chełm, ul. Pocztowa 54, 22-100 Chełm, Poland \\ e-mail: rogrodow@pwsz.chelm.pl \\ 2 Institute of Physics, UMCS, ul. Radziszewskiego 10, 20-031 Lublin, Poland \\ Received 11 May 2006 / Accepted 15 September 2006
}

\section{ABSTRACT}

\begin{abstract}
Aims. We investigate impulsively excited magnetosonic waves in a finely structured coronal loop that is modeled by a system of two parallel strands.

Methods. The equilibrium model is described by the time-dependent ideal magnetodydrodynamic equations that are solved numerically.

Results. Numerical simulations reveal that signatures of these waves in wavelet spectra depend on the distance between the strands and on the position of the initial pulse. In all cases, these spectra display complex structures that correspond to shortand long-period waves. We find that, with increasing distance between the strands, short-period waves dominate the wavelet spectra.

Conclusions. We demonstrate that the presence of a second strand affects wave propagation compared with one strand alone.
\end{abstract}

Key words. Sun: oscillations - Sun: magnetic fields - Sun: corona - magnetohydrodynamics (MHD)

\section{Introduction}

It has long been established both on theoretical and observational grounds that magnetohydrodynamic (MHD) waves can be sustained by solar coronal loops (e.g., Roberts et al. 1984; Aschwanden 1987). The recent interest in wave propagation in solar coronal loops (see, for example, Cooper et al. 2003; Nakariakov et al. 2004a; Diáz et al. 2004), spurred on by SOHO and TRACE observations (Nakariakov \& Ofman 2001; Aschwanden \& Nightingale 2005; Aschwanden 2005), has highlighted the complexity of developing of realistic models (e.g., Selwa et al. 2005; Verwichte et al. 2006; Diáz et al. 2006). The usual approach is to treat a coronal loop as a homogeneous magnetic column of condensed plasma (Diáz et al. 2004). Such an approach leads to dispersion relations that describe the normal modes of the system (Edwin \& Roberts 1982; Edwin 1991) and numerical solutions of the full set of magnetohydrodynamic (MHD) equations (e.g., Selwa \& Murawski 2004; Brady \& Arber 2005; Ofman 2005).

Solar coronal loops support both propagating and standing MHD waves. The standing waves are transverse (Aschwanden et al. 1999), vertical global kinks (Wang \& Solanki 2004) and sausage mode oscillations (Nakariakov et al. 2003). Standing slow waves are discussed by Ofman \& Wang (2002), Nakariakov et al. (2004b), Selwa et al. (2005), Taroyan et al. (2005), and Zaqarashvili et al. (2006). Propagating slow magnetosonic waves were detected near the footpoints of coronal loops (Berghmans \& Clette 1999; De Moortel et al. 2000). The fast-standing magnetosonic waves were recently studied by Nakariakov \& Verwichte (2005), Murawski et al. (2005), Verwichte et al. (2006) and
Diáz et al. (2006). Erdélyi \& Carter (2006a) and Erdélyi \& Fedun (2006b) investigated slow sausage surface waves in terms of solitons that propagate along magnetic flux structures. Propagating, fast magnetosonic waves in monolithic coronal loops were recently studied by, e.g., Nakariakov \& Ofman (2001), Nakariakov et al. (2004a), and Ogrodowczyk \& Murawski (2006).

Studies of composite coronal loops in the context of waves are rare. It was suggested by Nightingale et al. (2000) that a coronal loop consists of multi-threads or fibrils. We mention the early work of Murawski (1993), which proved by numerical simulations that the presence of two parallel slabs significantly affects wave propagation. An interaction between oscillating fibrils in such a system was studied by Diáz et al. (2004), who showed that wave frequencies of fast magnetosonic modes are the same in identical fibrils.

Recent analysis of TRACE data supports the idea of multistranded coronal structures (Aschwanden \& Nightingale 2005; Aschwanden 2005). It was found that elementary loops are nearly isothermal over their cross-sections and that the resolved loop structures could be composed of up-to $10 \mathrm{sub}-$ resolution structures. While analytical studies of waves in such complex structures are difficult, the system is amenable to numerical analysis.

The main purpose of this paper is to establish the influence of the composite structure of a coronal loop on propagating wave trains. We adopt a slab structure as a simple model of a loop strand. For a corona with a low filling factor there may be tens, if not hundreds or thousands, of strands comprising a loop such as observed by SOHO and TRACE. We perform parametric studies that allow us to determine the 


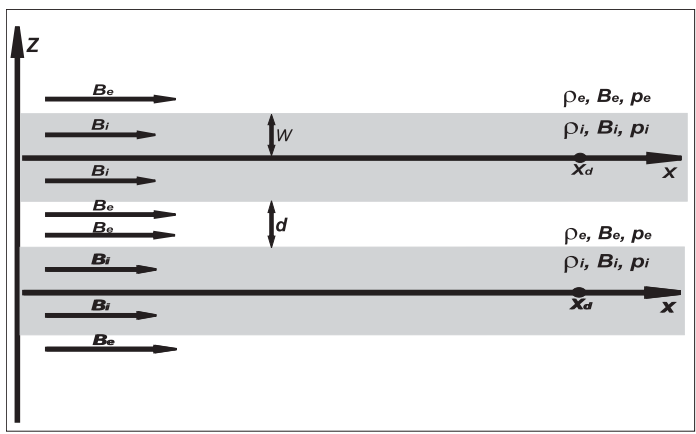

Fig. 1. The geometry of the model. The symbol $X_{\mathrm{d}}$ denotes the position of the detector and $d$ is the distance between the strands. Shaded regions correspond to the plasma slabs of enhanced mass density, with half-width $w$.

influence of the second strand on wave propagation in a loop consisting of two strands.

This paper is organized as follows. Sections 2 and 3 describe the physical and numerical models, respectively. Results of numerical simulations are presented in Sect. 4. This paper is concluded by a summary of the main results in Sect. 5.

\section{Physical model}

We consider an ideal, perfectly conducting, compressible plasma. Such plasma can be described by the time-dependent ideal MHD equations:

$$
\begin{aligned}
& \frac{\partial \varrho}{\partial t}+\nabla \cdot(\varrho \boldsymbol{V})=0, \\
& \varrho \frac{\partial \boldsymbol{V}}{\partial t}+\varrho(\boldsymbol{V} \cdot \nabla) \boldsymbol{V}=-\nabla p+\frac{1}{\mu}(\nabla \times \boldsymbol{B}) \times \boldsymbol{B}, \\
& \frac{\partial p}{\partial t}+\nabla \cdot(p \boldsymbol{V})=(1-\gamma) p \nabla \cdot \boldsymbol{V}, \\
& \frac{\partial \boldsymbol{B}}{\partial t}=\nabla \times(\boldsymbol{V} \times \boldsymbol{B}), \\
& \nabla \cdot \boldsymbol{B}=0 .
\end{aligned}
$$

Here $\varrho$ is mass density, $\boldsymbol{V}$ flow velocity, $\boldsymbol{B}$ the magnetic field, $p$ gas pressure, $\mu$ the magnetic permeability, and $\gamma=5 / 3$ is the adiabatic index.

Henceforth, we limit our discussion to a two-dimensional system for which the plasma quantities are independent of the spatial coordinate $y$, i.e. $\partial / \partial y=0$, and $\boldsymbol{V}_{y}=\boldsymbol{B}_{y}=0$. While a recently observed loop was shown to be composed of 10 strands (Aschwanden 2005), we consider a simple model in which a loop consists of two parallel and identical strands. These strands are separated by a distance $d$ (Fig. 1). The halfwidth of a single strand is denoted by $w$. We assume that all plasma quantities experience a sudden jump from their constant values in the ambient medium to their constant values inside each strand. The ambient/external values are denoted by the subscript e, while the internal quantities are denoted by the subscript i (Fig. 1).

We define a sound speed and the Alfvén speed in the ambient medium, as $c_{\mathrm{se}}=\sqrt{\gamma p_{\mathrm{e}} / \varrho_{\mathrm{e}}}$ and $V_{\mathrm{Ae}}=\sqrt{B_{\mathrm{e}}^{2} / \mu \varrho_{\mathrm{e}}}$, respectively. The plasma $\beta$ in the ambient medium is expressed by: the ratio of Alfvén speeds, $v=V_{\mathrm{Ae}} / V_{\mathrm{Ai}}$; temperature
Table 1. Equilibrium parameters.

\begin{tabular}{cc}
\hline \hline Quantity & Value \\
\hline$V_{\mathrm{Ae}}$ & $1 \mathrm{Mm} \mathrm{s}^{-1}$ \\
$\beta$ & $0.52 \times 10^{-3}$ \\
$\varrho_{\mathrm{e}}$ & $10^{-15} \mathrm{~kg} \mathrm{~m}^{-3}$ \\
$T_{\mathrm{i}} / T_{\mathrm{e}}$ & 2 \\
$r$ & 10 \\
$v$ & $\sqrt{10.1}$ \\
$l$ & $1 \mathrm{Mm}$ \\
$w$ & $2 \mathrm{Mm}$ \\
\hline
\end{tabular}

ratio, $T_{\mathrm{i}} / T_{\mathrm{e}}$; and the mass density ratio $r=\varrho_{\mathrm{i}} / \varrho_{\mathrm{e}}$. It is given as

$\beta=\frac{p_{\mathrm{e}}}{B_{\mathrm{e}}^{2} /(2 \mu)}=\frac{1}{\frac{T_{\mathrm{i}}}{T_{\mathrm{e}}} r-1} \frac{v^{2}-r}{v^{2}}$.

Plasma quantities inside the strands are then expressed as

$\varrho_{\mathrm{i}}=r \varrho_{\mathrm{e}}, \quad B_{\mathrm{i}}=B_{\mathrm{e}} \frac{\sqrt{r}}{v}, \quad p_{\mathrm{i}}=p_{\mathrm{t}}-\frac{B_{\mathrm{i}}^{2}}{2 \mu}$.

Here the total pressure $p_{\mathrm{t}}$ is defined as the sum of the gas and magnetic pressures, viz.

$p_{\mathrm{t}}=p_{\mathrm{e}}+\frac{B_{\mathrm{e}}^{2}}{2 \mu}$.

Numerical values for these parameters are presented in Table 1.

Equations (1)-(5) can be linearized and then applied to a single slab to derive a dispersion relation (Edwin 1991). This dispersion relation describes two sets of modes, namely slow and fast magnetosonic waves. Slow magnetosonic waves propagate with phase speeds close to the sound speed. These waves are weakly dispersive. Fast magnetosonic modes have phase speeds in the range $V_{\mathrm{Ai}}<\omega / k<V_{\mathrm{Ae}}$ and are strongly dispersive in the long wavelength limit. These waves are divided into sausage waves, which are symmetric pulsations of the loop with its central axis remaining undisturbed, and kink waves, which involve lateral displacement of the loop while maintaining its cross-section and with the axis resembling a wriggling snake. For a cold plasma $\left(c_{\mathrm{se}}=c_{\mathrm{si}}=0\right)$, the dispersion relation has the form (Edwin 1991)

$\tan (n w)=m / n$,

for kink modes, and

$\tan (n w)=-n / m$,

for sausage modes. Here we have set

$m^{2}=k^{2}-\frac{w^{2}}{V_{\mathrm{Ae}}^{2}}, \quad n^{2}=\frac{w^{2}}{V_{\mathrm{Ai}}^{2}}-k^{2}$,

with $k$ being the wavenumber along the $x$-direction. Note that $m>0$ corresponds to non-leaky or ducted waves. The leaky waves carry off energy from the slab. This leakage then results in the coupling of the two slabs by the transport of energy between them via the ambient medium. To the best of our knowledge the dispersion relation for propagating fast magnetosonic waves trapped in a two-parallel-slabs structure 


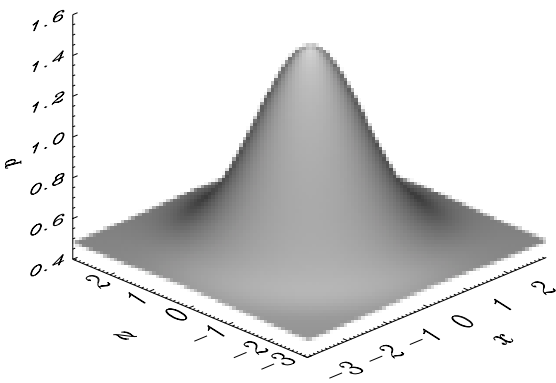

Fig. 2. The spatial profile of the initial pressure impulse, given by Eq. (12).

is unknown. One difficulty in the derivation of such a dispersion relation results from the coupling between these slabs. However, when these slabs become well-separated we expect that the coupling can be neglected and each slab oscillates independently.

\section{Numerical model}

We adopt the numerical code EMILY developed by Jones et al. (1997) to solve the time-dependent, non-ideal MHD equations, using a finite-volume scheme with an approximate Riemann solver for the hyperbolic fluxes, and central differencing schemes for the nested control volumes for the parabolic fluxes that arise from the non-ideal terms (i.e., resistivity and viscosity). The numerical scheme is accurate to the second order in space and time.

Equations (1)-(5) are solved numerically on a homogeneous grid with $600 \times 500$ cells. This grid covers a Eulerian box $(-30 l, 170 l) \times(-30 l, 30 l)$, where $l$ is a spatial unit of $1 \mathrm{Mm}$. Since our purpose is to study propagating waves, we used zero-gradient conditions at the boundaries of the simulation region to represent the natural extension of the real medium. As the numerical scheme is based on the method of characteristics, these boundary conditions work as transmitting boundaries that do not cause wave reflection back into the simulation domain. To perturb the system, we apply a pressure impulse of the form (see, Fig. 2)

$p(x, z, t=0)=p_{0}\left[1+A_{\mathrm{p}} \mathrm{e}^{-x^{2} / w_{\mathrm{p}}^{2}} \mathrm{e}^{-\left(z-z_{0}\right)^{2} / w_{\mathrm{p}}^{2}}\right]$,

where $p_{0}$ is the background pressure $\left(p_{\mathrm{e}}\right.$ or $\left.p_{\mathrm{i}}\right), A_{\mathrm{p}}$ denotes the relative amplitude of the pulse, $z_{0}$ is its initial position along the vertical direction, and $w_{\mathrm{p}}=2 w$ its width.

\section{Numerical results}

In this part of the paper we present results of the parametric studies we performed. We focus our attention on two parameters: (a) the distance between the strands, $d$; and (b) the impulse position, $z_{0}$. We perform wavelet and Fourier analyses, which allow us to determine the influence of the second strand on fast magnetosonic waves propagating in the system of two slabs.

\subsection{Pulse within the strand}

We launch the initial pulse inside the lower strand at $z_{0}=0$. Figure 3 shows spatial profiles of perturbed mass density

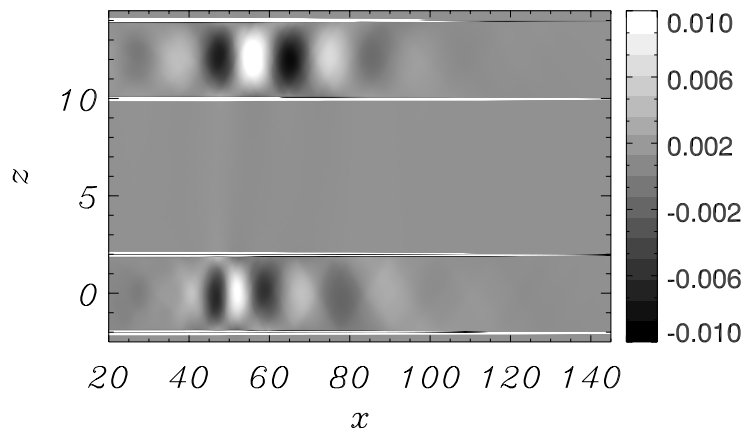

Fig. 3. Spatial profiles of perturbed mass density $\delta \varrho$, resulting from the initial pulse of Eq. (12) with $A_{\mathrm{p}}=2$ and $z_{0}=0$ at $t=370 \mathrm{~s}$. The distance between the strands is $d=4 \mathrm{w}$.

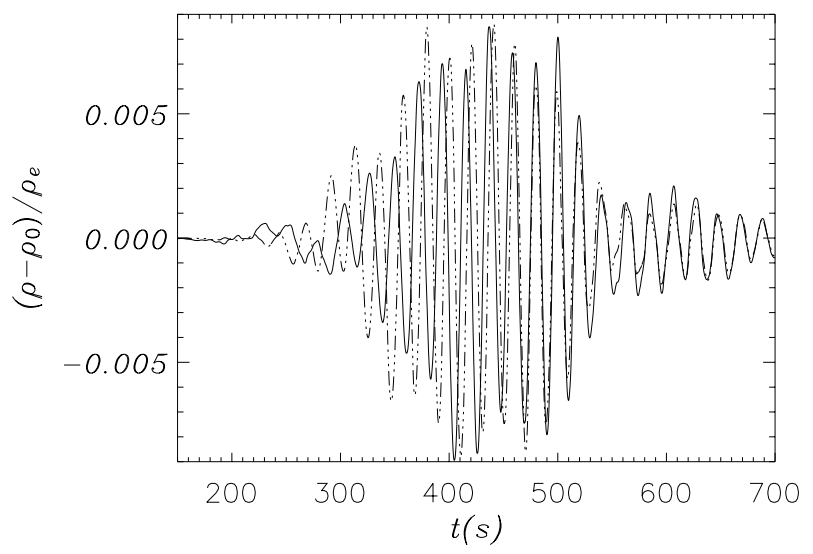

Fig. 4. Time signatures of the wave signals, collected at $x_{\mathrm{d}}=140 l$ inside the upper (dashed line) and lower (solid line) strands in the case of $d=4 w$.

$\delta \varrho=\varrho(x, z, t)-\varrho_{0}(z)$, in the two parallel slabs. Here $\varrho_{0}$ is the equilibrium mass density. We observe compressed plasma regions followed by rarified plasma and conclude that the initial pressure impulse excites sausage waves in the bottom strand. As a result of energy leakage from this strand, waves are excited in the top slab. However, it is interesting that these excited waves are sausage modes and not kink modes. We would expect that a perturbation localized outside the top strand triggers kink modes. This scenario is determined by the coupling between waves in the bottom and top strands. This coupling leads to sausage modes in both strands. Obviously the interaction between sausage modes in the bottom and top strands is stronger than the interaction between sausage and kink modes. As further evidence of the coupling is the fact that at $t=370 \mathrm{~s}$ the waves in the top slab possess larger amplitudes than oscillations in the bottom slab (Fig. 3). This result is in general agreement with the findings of Murawski \& Roberts (1994).

The coupling is evident in time signatures of wave signals collected on the axis of each strand (Fig. 4). Though the wave amplitudes are of comparable magnitude, a phase-shift between maxima is discernible. In the top strand, maxima occur at $t \approx 370 \mathrm{~s}$ and $430 \mathrm{~s} \leq t \leq 470 \mathrm{~s}$, and in the bottom strand at $t \approx 400 \mathrm{~s}$. These maxima are correlated. Wavelet (middle panel of Fig. 5) and Fourier (Fig. 6) spectra reveal two wave bands: the dominant wave period (sausage mode), $P \approx 22 \mathrm{~s}$, and the less pronounced wave period, $P \approx 60-80 \mathrm{~s}$ (sausage $(P \approx 63 \mathrm{~s})$ and kink $(P \approx 73 \mathrm{~s})$ mode $)$. Indeed, 

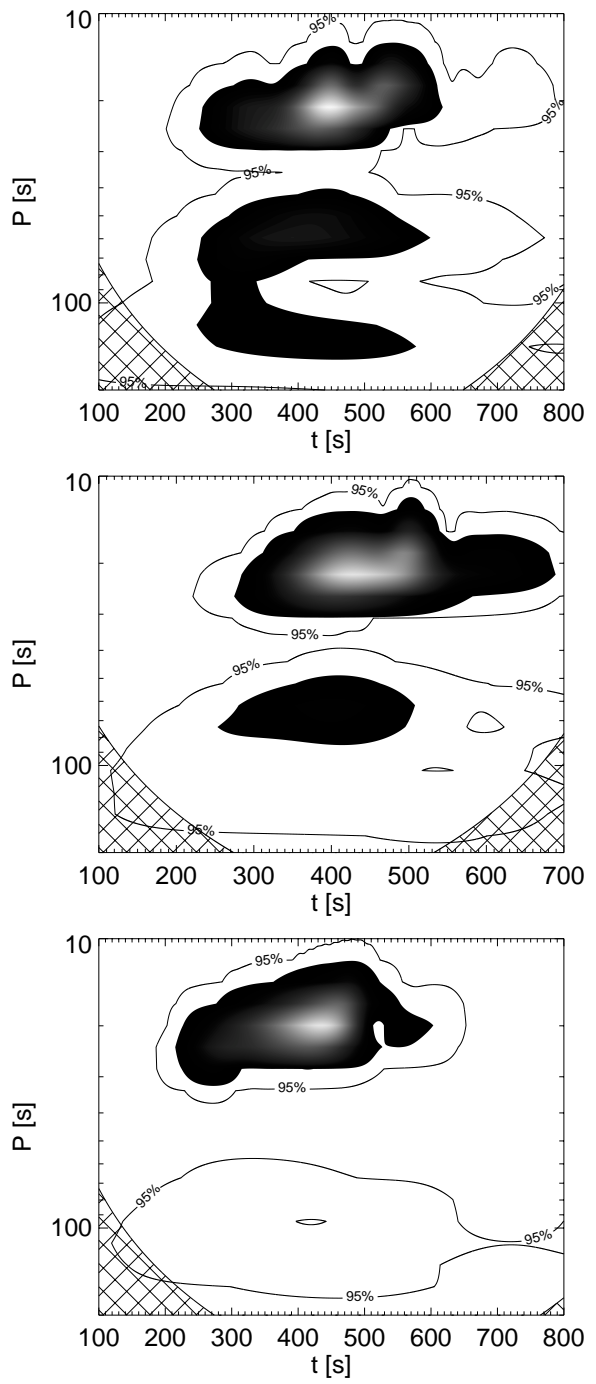

Fig. 5. Wavelet spectra of the wave signals, collected at $x_{\mathrm{d}}=140 \mathrm{l}$, for the initial pulse launched at $z_{0}=0$. The distance between the strands is $d=2 w$ (top panel), $d=4 w$ (middle panel), and $d=8 w$ (bottom panel). The bright (dark) patches correspond to the maximum (minimum) amplitude in the wavelet spectrum.

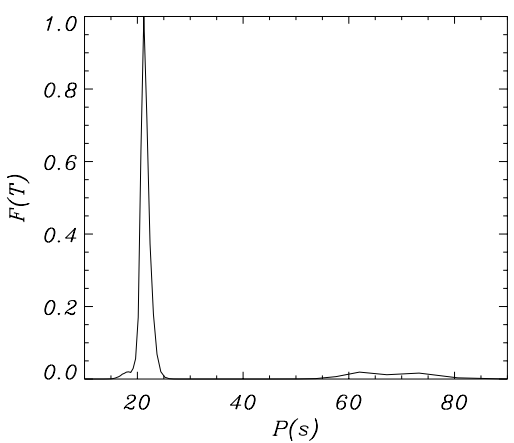

Fig. 6. Fourier spectrum of the wave signal of Fig. 4. The amplitude of the power spectrum $F(T)$ is normalized to 1 .

we can estimate the values of dominant wave periods from Fig. 6, which reveals the presence of new wave species in comparison to the single slab case (refer to Fig. 9 for comparison).
Table 2. Excited modes for the case of the initial pulse launched at $z_{0}=0$, with the corresonding wave frequencies as shown in the top panel of Fig. 11.

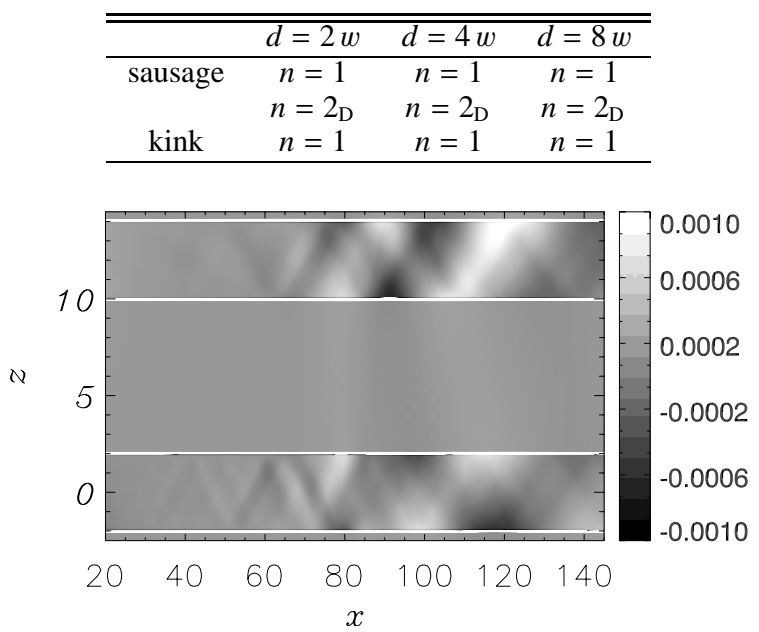

Fig. 7. Spatial profiles of perturbed mass density $\delta \varrho$ resulting from the initial pulse of Eq. (12) with $A_{\mathrm{p}}=2$ and $z_{0}=-5 l$ at $t=288 \mathrm{~s}$. The distance between the slabs is $d=4 w$.

Figure 5 shows wavelet spectra for different values of the distance between the strands: $d=2 w$ (top panel), $d=4 w$ (middle panel), and $d=8 w$ (bottom panel). The pressure impulse is launched at $z_{0}=0$. Longer wave periods appear in the wavelet spectra for low values of $d$. We conclude that for small distances between the strands, the effective half-width of the loop is increased and longer wavelength wave packets, comparable to this half-width, are firmly trapped by the system. It is clear that the sausage mode of $P \approx 22 \mathrm{~s}$ becomes dominant as $d \rightarrow \infty$. The time signatures and corresponding wavelet and Fourier spectra are then similar to the results for the single slab case (Ogrodowczyk \& Murawski 2006, top panels of their Fig. 3).

Table 2 summarizes the type and order of the modes that are excited by the impulse that is located within the lower strand $\left(z_{0}=0\right)$. Note that the subscript D denotes the dominant mode. In all these cases, the $n=2$ sausage mode is dominant although, the $n=1$ kink and sausage modes are also detected in the system.

\subsection{Pulse outside the strand}

To excite kink waves the pressure impulse was positioned outside the slab at $z_{0}=-5 l$ and $z_{0}=6 l$. First, we consider the distance between the strands $d=4 w$. Figure 7 shows the corresponding spatial profiles of perturbed mass density $\delta \varrho$, for the $z_{0}=-5 l$ case. We find that the impulse excites kink and sausage modes in both strands (see also the middle and bottom panels of Fig. 10). For $z_{0}=6 l$ the initial impulse launched between the strands excites the dominant kink and sausage modes (Fig. 8). This scenario is governed by the coupling between the bottom and top strands. As a consequence of this coupling, trapped sausage modes are excited in both strands, although the kink modes remain dominant. In the case of the initial impulse launched between the strands, Fourier amplitudes associated with the sausage modes are of comparable magnitude to kink modes (Fig. 8). We conclude 

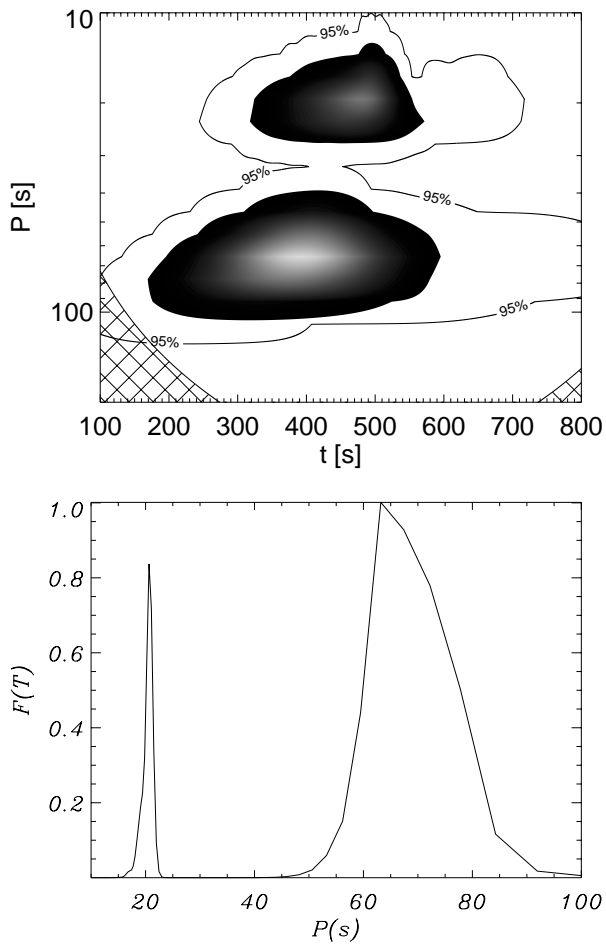

Fig. 8. Wavelet (top panel) and Fourier (bottom panel) spectra of the wave signals for the initial pulse launched at $z_{0}=6 l$ and the distance between the strands is $d=4 w$. The bright (dark) regions correspond to the maximum (minimum) amplitude in the wavelet spectrum. The amplitude of the Fourier spectrum is normalized to 1.

Table 3. Excited modes for $z_{0}=-5 l$ and $z_{0}=6 l$, with the corresponding wave frequencies as shown in middle and bottom panels of Fig. 11.

\begin{tabular}{ccccc}
\hline \hline & & $d=2 w$ & $d=4 w$ & $d=8 w$ \\
\hline$z_{0}=-5 l$ & kink: & $n=1_{\mathrm{D}}$ & $n=1_{\mathrm{D}}$ & $n=1_{\mathrm{D}}$ \\
& & $n=3$ & $n=3$ & $n=3$ \\
& sausage: & $n=1$ & $n=1$ & $n=1$ \\
& & $n=2$ & $n=2$ & \\
\hline$z_{0}=6 l$ & kink: & & $n=1_{\mathrm{D}}$ & $n=1_{\mathrm{D}}$ \\
& sausage: & $n=1_{\mathrm{D}}$ & $n=2$ & $n=2$ \\
\hline
\end{tabular}

that the presence of the second strand results in the excitation of sausage modes in the system.

Summary of the results that were obtained for various $z_{0}$ and $d$ are presented in Table 3 . The subscript $\mathrm{D}$ denotes a dominant mode, and the corresponding wave frequencies can be found in middle and bottom panels of Fig. 11. It is noteworthy that only the sausage mode is excited for $z_{0}=0$ and $d=2 w$. It agrees with top panel of Fig. 9. We infer that the strands are so close to each other that they act together so they can be considered as one single slab.

\subsection{Mode identification}

In this part of the paper we direct our efforts to identifying the modes excited in the system. However, the dispersion relation for the propagating fast magnetosonic waves in a twoparallel-slabs structure is unknown. Therefore, we adopt the dispersion relations for a single magnetic slab (Edwin 1991);
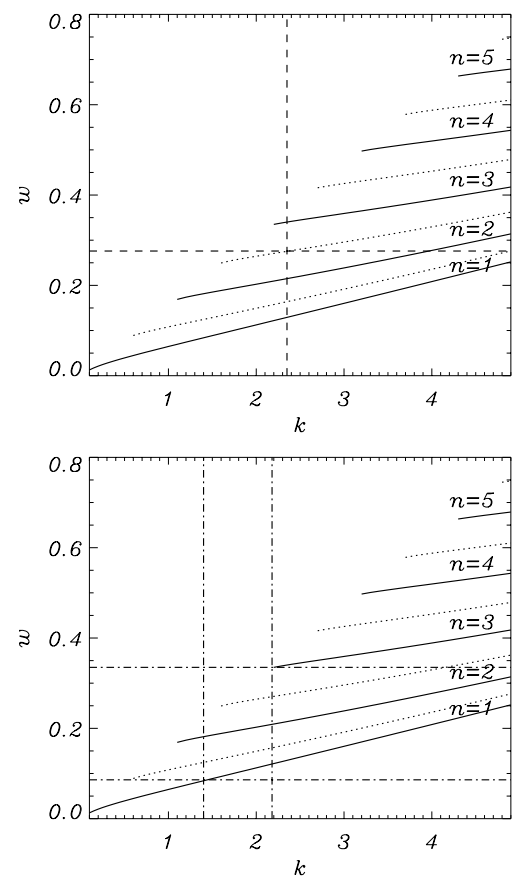

Fig. 9. Dispersive curves for fast magnetosonic waves in the case of $d \rightarrow \infty$. Solid (dotted) lines represent kink (sausage) modes. Dashed and dashed-dotted lines correspond to the case of the initial pulse launched at $z_{0}=0$ (top panel) and $z_{0}=-5 l$ (bottom panel). The number near each line denotes the order of the mode.

see Eqs. (10)-(11). We expect that when the slabs become well-separated, the coupling will be relatively small and our approach should reveal the modes present in a single slab. Any additional modes will then be due to the second slab.

Figure 9 displays wave frequency $\omega$ versus horizontal wavenumber $k$, which follows from the dispersion relations of Eqs. (10)-(11). The first-order $(n=1)$ kink mode is a ducted wave for all values of $k$. The higher-order modes exist as trapped waves for sufficiently high values of $k$. Long waves are called leaky as they propagate in the ambient medium and transfer energy from a slab to its neighborhood. We consider the case of the bottom slab alone $(d \rightarrow \infty)$ and launch the impulse at $z_{0}=0$. This impulse excites the second-order $(n=2)$ sausage modes in the slab (top panel of Fig. 9). Moving this pulse to $z_{0}=-5 l$ triggers the $n=1$ and $n=3$ kink modes.

Figure 10 illustrates dispersive curves for fast magnetosonic waves propagating along a single slab, in the cold plasma approximation. This strand is positioned at the distance $d=4 w$ from the top strand. The top (middle) panel of Fig. 10 should be compared with the top (bottom) panel of Fig. 9. The second-order sausage mode is present both in the single strand alone (top panel of Fig. 9) and in the system of two strands (top panel of Fig. 10). Due to the presence of the top strand, two additional modes are triggered in the bottom strand. They are identified as the $n=1$ sausage and $n=1$ kink modes. When the pressure impulse is launched below the bottom slab at $z_{0}=-5 l$ (middle panel of Fig. 10), the $n=1$ and $n=2$ sausage modes are excited in addition to the modes presented for a single slab (Fig. 9). The presence of these modes serves as evidence of the coupling between the strands. When the initial pulse is launched in the region between two strands, at $z_{0}=6 l$, the $n=1 \mathrm{kink}$ and $n=2$ sausage modes are excited. Although the presence of 

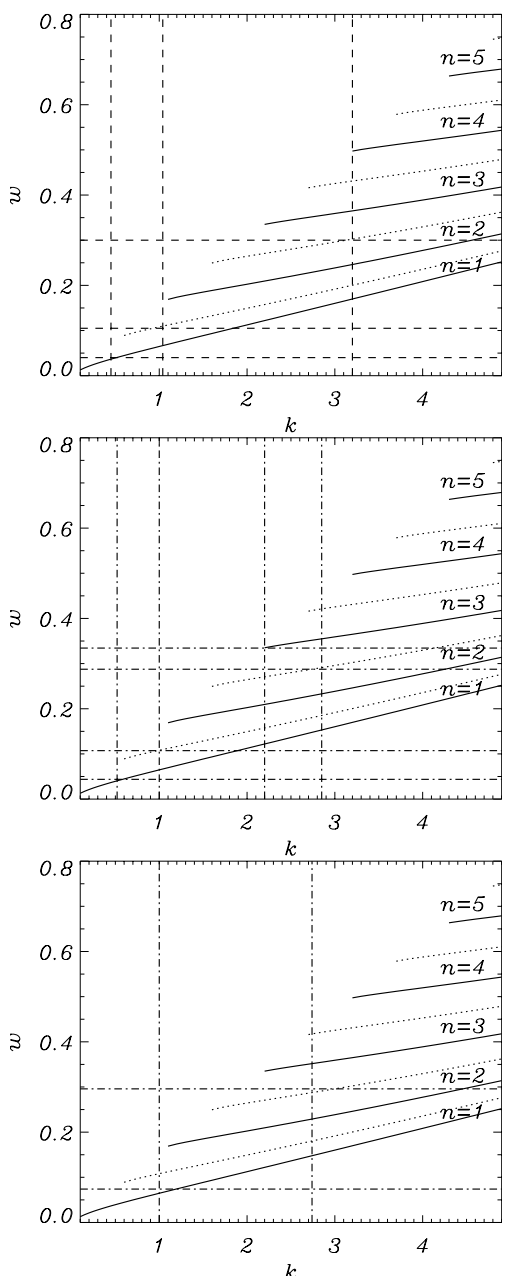

Fig. 10. Analytically evaluated dispersive curves for fast magnetosonic waves. Solid lines represent kink modes and dotted lines correspond to sausage modes for a single, cold $(\beta=0)$ plasma strand. Dashed and dashed-dotted lines correspond to $\omega$ and $k$ evaluated for the case of two strands separated by a distance $d=8 l$. The initial pulse launched at $z_{0}=0$ (top panel), $z_{0}=-5 l$ (middle panel) and $z_{0}=6 l$ (bottom panel). The number near each line denotes the order of the mode.

the kink mode is not surprising, the sausage mode seems to be a result of the coupling. We infer that the trapped sausage modes in both strands interact with each other via the ambient medium. Such influences amplify these modes and their amplitudes become comparable with kink oscillations. Note that the sausage waves are not observed in the single slab.

\subsection{Wave frequency vs. distance between the slabs}

In this part of the paper we discuss the influence of the distance between the slabs on spectral properties of the excited waves. Figure 11 displays wave frequency $\omega$ versus normalized distance, $d / w$, between the strands. The top panel shows $\omega$ for the initial pulse launched at $z_{0}=0$. It is clear that $\omega$ is lower for higher values of $d / w$. We conclude that kink modes result from the presence of the second strand in the system. The wave signal that reaches the top strand is reflected and excites the kink oscillations in the same manner as the impulse located in the bottom slab. The middle
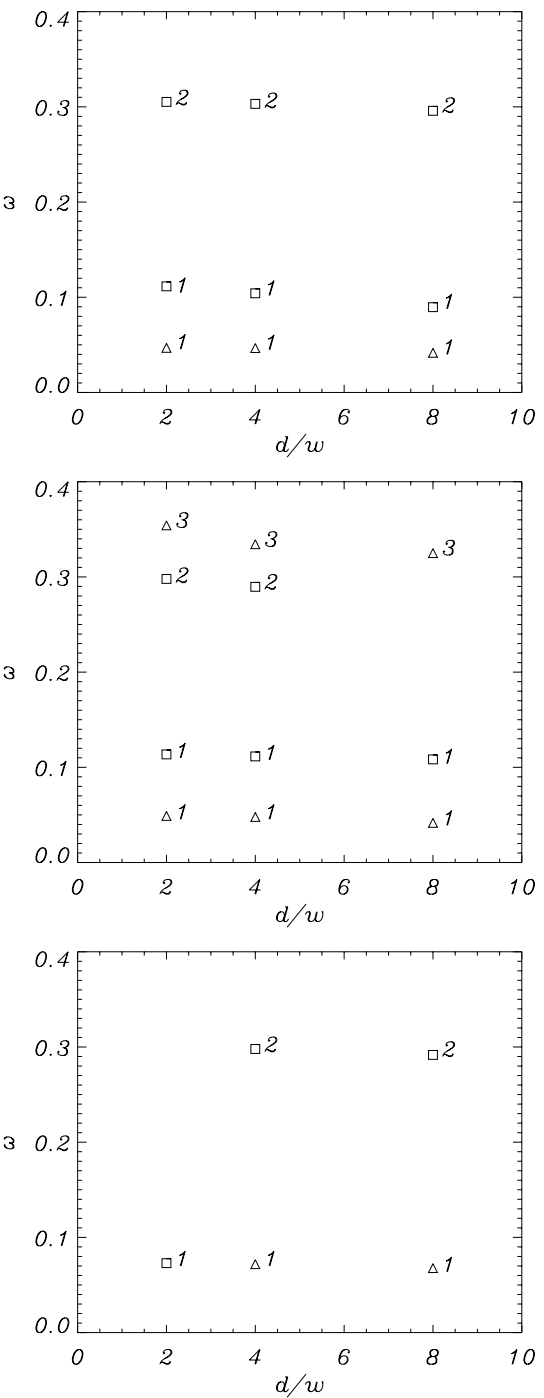

Fig. 11. Wave frequency $\omega$ vs. normalized distance $d / w$ between the slabs. The initial pulse is launched at $z_{0}=0$ (top panel), $z_{0}=-5 l$ (middle panel), and $z_{0}=w+d / 2$ (bottom panel). The triangles (squares) correspond to kink (sausage) modes. The number near the symbol denotes the order of the mode.

panel of Fig. 11 displays $\omega$ for the initial position of the pulse at $z_{0}=-5 l$. The impulse located below the system of strands excites the sausage and kink modes. We conclude that this scenario is affected by the coupling between the strands. It is noteworthy that only one sausage mode is excited for $d=8 w=16 l$. For a lower value of $d / w$, two sausage modes are excited. The bottom panel of Fig. 11 corresponds to the case when the impulse is launched exactly between slabs $\left(z_{0}=w+d / 2\right)$. The first- and second-order sausage and kink waves are excited, depending on the distance between two slabs. We conclude that the interaction between waves trapped in each slab decreases with $d$. Note that the number of excited sausage modes declines with increasing $d$ and the kink mode becomes dominant instead (middle and bottom panel of Fig. 11). It is noteworthy that $\omega$ decreases for higher values of $d / w$. We infer that for $d \rightarrow \infty$ the coupling between the slabs is smaller, which results from how waves propagating in the ambient medium do not reach the other 
slab. Longer wave periods are excited in such systems because they are not so frequently affected by the perturbations.

\section{Summary}

In this paper we performed numerical simulations of impulsively generated magnetosonic waves in a coronal loop that consists of two parallel slabs. The results of these simulations reveal that kink and sausage waves possess properties that depend on the distance between the slabs. We find out that the coupling between the strands influences excitation of sausage modes when the initial pulse is launched outside the strands. As a result of this coupling, amplitudes of oscillations vary interchangeably in both strands. We identified the modes excited in the system. In comparison to the case of a single slab, additional kink and sausage modes are triggered in both strands. The parametric studies we performed reveal that the wave frequency, $\omega$, is smaller for higher values of the distance between the strands, $d$. The strength of the coupling between the strands decreases with increasing $d$. It is noteworthy that a smaller number of sausage modes are excited for higher values of $d$. On the other hand, it is discernible that larger wave periods, $P$, are present in the wavelet spectra for lower $d$. We conclude that for a small distance between the strands the effective half-width is increased and wave packets with longer wave period are ducted along the structure.

Acknowledgements. The authors express their cordial thanks to an unknown referee for his/her comments that helped to improve this paper. K.M.'s work was financially supported by a grant from the State Committee for Scientific Research Republic of Poland, with KBN grant No. 2PO3D 016 25. The magnetohydrodynamics code used in this study was developed at the University of Washington by Ogden S. Jones, Uri Shumlak, Scott Eberhardt, Bogdan Udrea, and was provided through the sponsorship of AFOSR program. The wavelet software was written by C. Torrence and G. Compo, and is available at URL: http: //paos. colorado.edu/research/wavelets

\section{References}

Aschwanden, M. J. 1987, Sol. Phys., 111, 113

Aschwanden, M. J. 2005, ApJ, 634, 193

Aschwanden, M. J., \& Nightingale, R. W. 2005, ApJ, 633, 499

Aschwanden, M. J., Fletcher, L., Schrijver, C. J., \& Alexander, D. 1999, ApJ, 520, 880

Berghmans, D., \& Clette, F. 1999, Sol. Phys., 186, 207

Brady, C. S., \& Arber, T. D. 2005, A\&A, 438, 733

Cooper, F. C., Nakariakov, V. M., \& Williams, D. R. 2003, A\&A, 409, 325

De Moortel, I., Ireland, J., \& Walsh, R. W. 2000, A\&A, 355, L23

Diáz, A. J., Oliver, R., Ballester, J. L., \& Roberts, B. 2004, A\&A, 424, 1055

Diáz, A. J., Zaqarashvili, T. V., \& Roberts, B. 2006, A\&A, 455, 709

Edwin, P. M. 1991, Ann. Geophys., 9, 188

Edwin, P. M., \& Roberts, B. 1982, Sol. Phys., 76, 239

Erdélyi, R., \& Carter, B. K. 2006, A\&A, 455, 361

Erdélyi, R., \& Fedun, V. 2006, Phys. Plasmas, 13, 032902

Jones, O. S., Shumlak, U., \& Eberhardt, D. S. 1997, J. Comput. Phys., 130, 231

Murawski, K. 1993, Acta Astron., 43, 161

Murawski, K., \& Roberts, B. 1994, Sol. Phys., 151, 305

Murawski, K., Selwa, M., \& Rossmanith, J. A. 2005, Sol. Phys., 231, 87

Nakariakov, V. M., \& Ofman, L. 2001, A\&A, 372, L53

Nakariakov, V. M., \& Verwichte, E. 2005, Liv. Rev. Sol. Phys., 2, 3

Nakariakov, V. M., Melnikov, V. F., \& Reznikova, V. E. 2003, A\&A, 412, L7

Nakariakov, V. M., Tsiklauri, D., Kelly, A., Arber, T. D., \& Aschwanden, M. J. 2004a, A\&A, 414, L25

Nakariakov, V. M., Arber, T. D., Ault, C. E., et al. 2004b, MNRAS, 349, 705 Nightingale, R. W., Aschwanden, M. J., Alexander, D., Reale, F., \& Peres, G. 2000, A\&AS, 32, 812

Ofman, L. 2005, Adv. Space Res., 36, 1572

Ofman, L., \& Wang, T. 2002, ApJ, 580, L85

Ogrodowczyk, R., \& Murawski, K. 2006, Sol. Phys., 236, 273

Roberts, B., Edwin, P. M., \& Benz, A. O. 1984, ApJ, 279, 857

Selwa, M., \& Murawski, K. 2004, A\&A, 425, 719

Selwa, M., Murawski, K., \& Solanki, S. K. 2005, A\&A, 437, 701

Taroyan, Y., Erdélyi, R., Doyle, J. G., \& Bradshaw, S. J. 2005, A\&A, 438, 713

Verwichte, E., Foullon, C., \& Nakariakov, V. M. 2006, A\&A, 446, 1139

Wang, T. J., \& Solanki, S. K. 2004, A\&A, 421, L33

Zaqarashvili, T. V., Oliver, R., \& Ballester, J. L. 2006, A\&A, 456, L13 